\title{
Cetoacidose diabética canina
}

\author{
Canine ketoacidosis \\ Leandro Zuccolotto Crivelenti ${ }^{\mathrm{I}}$ Sofia Borin' ${ }^{\mathrm{I}}$ Alexandre Martini de Brum${ }^{\mathrm{II}}$ \\ Mirela Tinucci-Costa ${ }^{\text {III }}$
}

RESUMO

A cetoacidose diabética (CAD), uma das complicações metabólicas em pacientes com diabetes mellitus (DM), caracteriza-se por hipercetonemia e alterações do equilíbrio ácido-base, juntamente com as alterações clínicas $e$ laboratoriais compatíveis. Este trabalho objetiva apresentar uma revisão crítica dos principais pontos da etiopatogenia, dos sinais clínicos e das alterações laboratoriais da CAD, bem como discorrer sobre prognóstico e modalidades terapêuticas mais recentes, visando a fornecer subsídios ao clínico de pequenos animais.

Palavras-chave: cães, endocrinopatia, hiperglicemia, hipercetonemia, acidose metabólica.

\section{ABSTRACT}

The diabetic ketoacidosis (DKA), one of the metabolic complications in patients with diabetes mellitus (DM), is characterized by hiperketonemia and acid-basic disturbances, in association with suitable clinic and laboratorial changes. This research aims to present a critical review on the main aspects of ethiopathogenesis, clinical sings and laboratorial changes of DKA, as well as the prognosis and the most recent therapeutic manners, in order to provide assistance to the small animal clinic.

Key words: dogs, endocrinopathy, hyperglycemia, hiperketonemia, metabolic acidosis.

\section{INTRODUÇÃO}

A cetoacidose diabética (CAD) trata-se de uma desordem metabólica aguda, potencialmente fatal, tanto em humanos, quanto em caninos com diabetes mellitus (DM) (BRUYETTE, 1997; DUARTE et al., 2002; BOYSEN, 2008; DI TOMMASO et al., 2009). A prevalência de DM na população canina tem aumentado consideravelmente nos últimos anos, sendo atribuída às mudanças comportamentais associadas ao sedentarismo, à obesidade e ao estresse (PÖPPL \& GONZÁLEZ, 2005). Estudos apontam que, de cada 200 cães diabéticos, um desenvolve CAD, enquanto que nos gatos essa proporção é de 800 para 1 (BRUYETTE, 1997).

A história da CAD mostra que praticamente todos os animais morriam antes do advento da insulinoterapia na medicina veterinária (BRUYETTE, 1997), contudo esse panorama se modificou. A conscientização de que a CAD é uma emergência e necessita de rápido diagnóstico e tratamento diminuiu consideravelmente o índice de mortalidade (DUARTE et al., 2002).

A CAD está associada, preferencialmente, aos caninos diabéticos insulino-dependentes (CHASTAIN, 1981; HUME et al., 2006), com idade entre

\footnotetext{
IPrograma de Pós-graduação em Medicina Veterinária, Faculdade de Ciências Agrárias e Veterinárias (FCAV), Universidade Estadual Paulista (UNESP), 14870-000, Jaboticabal, SP, Brasil. E-mail: crivelenti_lz@yahoo.com.br. *Autor para correspondência.

IIDepartamento de Clínica Médica de Pequenos Animais, Universidade de Franca (UNIFRAN), SP, Brasil.

IIIDepartamento de Clínica e Cirurgia Veterinária, FCAV, UNESP, Jaboticabal, SP, Brasil.
} 
cinco e 12 anos de idade, diagnosticados entre os oito a nove anos, em média (DUARTE et al., 2002; HUME et al., 2006), sendo duas vezes mais frequente nas fêmeas que em machos (DE MARCO et al., 1999; DUARTE et al., 2002). Um estudo retrospectivo feito por HUME et al. (2006) mostrou que, de 127 caninos cetoacidóticos, Poodle miniatura e toy (16\%), Rottweiler (6\%) e Yorkshire Terrier (5\%) foram os mais acometidos. No entanto, a maioria era constituída de caninos sem raça definida (21\%).

\section{Etiofisiopatogenia}

A deficiência insulínica grave ou absoluta suscita o aumento dos hormônios contra-reguladores (glucagon, hormônio do crescimento, glicocorticóides e catecolaminas), potencializando a atividade cetogênica (FOSS-FREITAS \& FOSS, 2003), com a produção de corpos cetônicos que excedem a capacidade do organismo em transformá-los em energia (CHASTAIN, 1981). Os corpos cetônicos são formados no fígado a partir da metabolização de ácidos graxos livres decorrentes da lipólise em ácido ß-hidroxibutírico (ßHB), gerando ácido acetoacético (AcAc) e acetona (CHASTAIN, 1981; BOYSEN, 2008; DI TOMMASO et al., 2009).

A causa da acidose é multifatorial; no entanto, o maior componente está na produção dos ácidos ß-hidroxibutírico e acetoacético. O AcAc é a primeira cetona produzida pelo fígado, a qual é reduzida em ßHB ou decarboxilada em acetona. Esta última é quimicamente neutra, mas as outras duas cetonas são ácidos orgânicos (BOYSEN, 2008). O acúmulo desses compostos resulta em acidose metabólica, com consequente diminuição do $\mathrm{pH}$ e da concentração do bicarbonato arterial (DI TOMMASO et al., 2009). Ademais, tais cetonas são filtradas pelos rins e parcialmente excretadas na urina, causando cetonúria e contribuindo para a diurese osmótica (BOYSEN, 2008).

Resumidamente, a inabilidade na utilização da glicose induz o catabolismo das reservas corporais (GRECO, 2001; NELSON, 2006), o aumento da cetogênese hepática, da lipólise e a mobilização de ácidos graxos livres, culminando em hipercetonemia e alterações do equilíbrio ácido-base (FOSTER \& McGARRY, 1983; FOSS-FREITAS \& FOSS, 2003).

Um fator importante a ser considerado no desenvolvimento da CAD é a hipovolemia, uma vez que, quando a glicose excede o limiar tubular renal de absorção, segue-se a diurese osmótica, com perda de glicose, eletrólitos e água. O córtex renal é especialmente susceptível às toxinas e à hipovolemia que interrompem as vias metabólicas geradoras de ATP. A isquemia pode, rapidamente, esgotar os estoques de energia das células, levando à depleção das reservas energéticas. Com isso, ocorre falha da bomba $\mathrm{Na}+/ \mathrm{K}+$-ATPase, levando a edema e à morte celular (NELSON, 2006). Perdas gastrintestinais decorrentes de vômito e diarreia são frequentemente reportadas como sinais clínicos da CAD e contribuem com a hipovolemia desenvolvida nos pacientes (BOYSEN, 2008).

A diminuição da perfusão renal acarretará redução da taxa de filtração glomerular e azotemia; porém, se esta permanecer por tempo prolongado, pode resultar em necrose tubular aguda isquêmica e, consequentemente, em insuficiência renal aguda e anúria (CHASTAIN, 1981), que constitui grave complicação da CAD, dificultando o tratamento e piorando o prognóstico dos pacientes. Os aumentos de concentrações séricas de creatinina e ureia indicam prejuízo na função renal, podendo ter origens, tanto pré, quanto renal (NELSON, 2006).

As concentrações dos eletrólitos plasmáticos também são alteradas na CAD. O sódio pode estar baixo, normal ou aumentado, mas a maioria dos estudos demonstrou sódio baixo no momento do diagnóstico. A mudança de compartimento do fósforo e potássio do meio extracelular para o intracelular na presença de acidose metabólica e hipoinsulinemia, associada à perda desses íons pelos rins em decorrência da diurese osmótica e deficiência insulínica (a insulina é necessária para reabsorção normal de sódio, potássio, fósforo e cloreto pelas células epiteliais tubulares renais), ocasionará hipocalemia e hipofosfatemia. Hipomagnesemia não é um achado comum; não obstante, a concentração de magnésio deve ser monitorada sempre que possível, na presença de arritmias e/ou hipocalemia ou hipocalcemia refratárias (BOYSEN, 2008).

Os fatores precipitantes da CAD podem ser de natureza infecciosa (respiratória, urinária, genital, cutânea, etc.) (FOSS-FREITAS \& FOSS, 2003) ou ser provocados por pancreatite aguda, administração de medicamentos diabetogênicos (glicocorticóides, acetato de megestrol), hiperadrenocorticismo, desidratação, anorexia (CHASTAIN, 1981), insulite imunomediada, amiloidose nas ilhotas pancreáticas (PÖPPL \& GONZÁLEZ, 2005) e tratamento insulínico interrompido ou inadequado às condições do paciente (DUARTE et al., 2002; FOSS-FREITAS \& FOSS, 2003).

\section{Sinais clínicos e alterações laboratoriais}

A inabilidade da produção e/ou utilização da insulina causa distúrbios na metabolização de carboidratos, lipídeos e proteínas (BUSH, 2004; NELSON, 2006), resultando, frequentemente, em quadro 
hiperglicêmico (McGUIRE, 2002), podendo piorar os sinais clássicos de poliúria/polidipsia (PU/PD), polifagia e perda de peso da DM (GRECO, 2001; McGUIRE et al., 2002; NELSON, 2006).

Com a progressão do quadro, o paciente pode manifestar anorexia, adipsia, depressão, vômitos, diarreia, taquipneia, dor, distensão abdominal (FELDMAN \& NELSON, 1996), desidratação, pirexia (CHASTAIN, 1981) e, em casos mais avançados, hálito cetônico, alterações respiratórias compensatórias (respiração de Kussmaul) e alterações no SNC, desde sonolência, torpor, confusão mental até estabelecimento de estado de coma profundo (FOSSFREITAS \& FOSS, 2003).

Nos resultados laboratoriais, há hiperglicemia (aproximadamente $500 \mathrm{mg} \mathrm{dL}^{-1}$ ) e hipercetonemia, na presença de acidose metabólica (BRUYETTE, 1997; DUARTE et al., 2002; UMPIERREZ et al., 2004b; BOYSEN, 2008; DI TOMMASO et al., 2009). Glicosúria e cetonúria são achados compatíveis, e a densidade urinária específica pode encontrar-se baixa pela diurese osmótica (PINEDA \& DOOLEY, 2003).

Em pequenos animais, o grau de cetonemia é tradicionalmente avaliado por testes semiquantitativos que estimam a concentração de AcAc na urina. Esses testes, baseados na reação com nitroprusside, não são capazes de detectar a presença de ßHB, o predominante corpo cetônico produzido na CAD. A razão ßHB:AcAc é de 3:1 em pacientes cetoacidóticos, chegando a razões de 20:1 em processos mais severos (DUARTE et al., 2002). Dessa forma, dois pontos negativos devem ser ressaltados em relação à dosagem de corpos cetônicos urinários: severa hipercetonemia pode ser subestimada ou não detectada (DI TOMMASO et al., 2009), assim como pacientes em cetose diabética, os quais não necessitariam de terapias agressivas, podem ser erroneamente diagnosticados como cetoacidóticos (DUARTE et al., 2002).

Por essas razões, métodos de dosagem de ßHB sanguíneo são, atualmente, preferidos aos testes urinários ou sanguíneos de detecção do AcAc para o diagnóstico e a monitoração da CAD em humanos (DI TOMMASO et al., 2009) e caninos (DUARTE et al., 2002). Estudo complementar realizado por DI TOMMASO et al. (2009) demonstrou eficiência equivalente nas dosagens sanguíneas de ßHB por meio de sensor portátil. Salienta-se, no entanto, que esse dado deve ser sempre associado aos sinais clínicos do paciente para se estabelecer o diagnóstico da CAD (DUARTE et al., 2002).

A elevação do hematócrito reflete o quadro de hemoconcentração e hipovolemia. Pode também haver aumento na concentração de colesterol, sendo resultado da acentuada lipólise. As concentrações séricas de sódio, potássio e fosfato mostram muitas variações e são dependentes, além dos fatores já descritos, de vômitos frequentes, ingestão hídrica reduzida pela depressão mental e anorexia (BRUYETTE, 1997).

A monitoração da CAD canina é, algumas vezes, dificultada, considerando-se a necessidade da mensuração, além da glicemia e de corpos cetônicos, de gases e de eletrólitos sanguíneos (DI TOMMASO et al., 2009). O hemogasômetro, aparelho utilizado para a monitoração, apresenta alto custo de aquisição e está disponível, na maioria das vezes, em centros de referência (CHASTAIN, 1981).

\section{Tratamento e prognóstico}

Os objetivos primários do tratamento da CAD são a restauração do volume intravascular, correção da desidratação e dos distúrbios eletrolítico e ácido-base, e redução das concentrações de glicose sanguínea (BOYSEN, 2008). O sucesso do tratamento dependente do estado clínico no momento do diagnóstico e da instituição de terapia apropriada às condições de cada paciente (CHASTAIN, 1981).

A fluidoterapia é um dos pontos-chave no tratamento da CAD, e as soluções de Ringer com Lactato e $\mathrm{NaCl}$ 0,9\% podem ser utilizadas, e a velocidade de infusão depende do grau de desidratação. Na ausência de insuficiência cardíaca ou insuficiência renal anúrica ou oligúrica, recomenda-se a administração de $15-20 \mathrm{~mL} \mathrm{~kg}^{-1} \mathrm{~h}^{-1}$ ou $20 \%$ do déficit de fluido calculado na primeira hora de terapia, seguido de $30 \%$ do cálculo nas próximas quatro a cinco horas. Os 50\% restantes devem ser administrados nas 18 horas seguintes, de modo que o déficit seja completamente corrigido em 24 horas (BOYSEN, 2008). Em casos de hiperosmolaridade, pode-se optar pela solução de $\mathrm{NaCl}$ a $0,45 \%$. Concentrações de glicose sérica superiores a $500 \mathrm{mg} \mathrm{dL}^{-1}$ representam alto potencial à insuficiência renal aguda em cães, fazendo-se necessário o emprego de fluidoterapia agressiva e monitoração do débito urinário (CHASTAIN, 1981). Fluidoterapia de manutenção deve ser instituída após a correção da hipovolemia, baseada no peso corpóreo e nas perdas futuras ([(Peso (kg) x 30) + 70]) (BOYSEN, 2008).

Outro ponto importante no tratamento da CAD é a terapia insulínica para redução da hiperglicemia, e o ideal é a utilização de insulinas de rápida ação. Nesse caso, a insulina regular (insulina $\mathrm{R}$ ) é a terapia de escolha. A meia-vida da insulina R, administrada pela via intramuscular, é de duas horas, portanto, baixas doses, assim como descrito em humanos (BARONE et al., 2007), podem ser usadas 
como método efetivo e seguro no tratamento da CAD canina. Em cães com pesos inferiores a 10kg, utilizamse doses iniciais de duas unidades e posteriores repetições de uma unidade a cada hora. Para cães com pesos superiores a $10 \mathrm{~kg}$, utilizam-se doses iniciais de $0,25 \mathrm{UI} \mathrm{kg}{ }^{-1}$ e sequenciais repetições de $0,1 \mathrm{UI} \mathrm{kg}^{-1}$ a cada hora (CHASTAIN \& NICHOLS, 1981). Para controle da efetividade da terapia, espera-se que a glicemia diminua, aproximadamente, $100 \mathrm{mg} \mathrm{dL}^{-1}$ após uma hora da aplicação de uma dose de insulina R. Se a redução glicêmica for muito inferior ao esperado, é necessário certificar-se da via de administração, da dose e se a insulina está viável. Cuidados adicionais devem ser tomados em animais muito obesos, pois pode haver demora na ação da insulina devido à dificuldade de acesso desta aos receptores. Quando a glicemia for reduzida a valores entre 150 e $250 \mathrm{mg} \mathrm{dL}^{-1}$, o regime de administração de insulina $\mathrm{R}$, a cada duas horas, é descontinuado, e uma solução de dextrose a 5\% com 4 a $8 \mathrm{mEq} \mathrm{L}{ }^{-1}$ de fosfato de potássio deve ser empregada, para evitar hipoglicemia e hipofosfatemia.

Outro protocolo de insulinoterapia em paciente com CAD sugere uma dose inicial de 0,2UI $\mathrm{kg}^{-1}$, via intramuscular, seguida de $0,1 \mathrm{UI} \mathrm{kg}^{-1}$ de hora em hora ou em infusões de baixas doses de 0,05 a 0,1UI $\mathrm{kg}^{-1} \mathrm{~h}^{-1}$, em solução de $\mathrm{NaCl}$ a $0,9 \%$, até se reduzir a glicemia a valores inferiores a $250 \mathrm{mg} \mathrm{dL}^{-1}$. Depois, a insulina regular deve ser administrada na dose de 0,1 a 0,4UI $\mathrm{kg}^{-1}$, via subcutânea, a cada seis a oito horas (NOGUEIRA \& DE MARCO, 2008). O tratamento da CAD com infusão contínua de baixas doses de insulina regular pela via intravascular é realizado com sucesso em humanos (UMPIERREZ et al., 2004a; BARONE et al., 2007) e caninos cetoacidóticos (HUME et al., 2006). O protocolo terapêutico para uso em cães foi proposto por MANCITIRE (1993), o qual consiste na adição de insulina R na dose de $2.2 \mathrm{UI} \mathrm{kg}^{-1}$ para 24 horas $(0,09 \mathrm{UI}$ $\mathrm{kg}^{-1} \mathrm{~h}^{-1}$ ), em $250 \mathrm{~mL}$ de solução de $\mathrm{NaCl} 0,9 \%$ ou de Ringer simples. Deve-se, no entanto, permitir que 50mL da diluição passe pelo equipo de administração, pois a insulina adere ao plástico do tubo. Dessa forma, saturam-se os sítios de adesão, permitindo-se saber com exatidão a quantidade de insulina a ser administrada por mL de solução. Com uso de bomba de infusão, deve-se administrar $10 \mathrm{~mL} \mathrm{~h}^{-1}$ da solução até que se obtenha concentração de glicose inferior ou igual a $250 \mathrm{mg} \mathrm{dL}^{-1}$. A partir de então, reduz-se a velocidade para $5-7 \mathrm{~mL} \mathrm{~h}^{-1}$ e acrescenta-se solução de glicose $5 \%$ até que sejam alcançados $100 \mathrm{mg} \mathrm{dL}^{-1}$ de glicemia e o animal volte a se alimentar espontaneamente.

Diversas pesquisas em humanos são voltadas a terapias com baixas doses de insulina regular pela via subcutânea (SC) como alternativa ao tratamento intravascular, com o objetivo de se obter maior segurança com redução dos custos e dos cuidados intensivos (UMPIERREZ et al., 2004a). Entretanto, essa via não deve utilizada no tratamento da CAD em cães, pois fatores como a desidratação e hipovolemia prejudicam a absorção da insulina e o controle do quadro hiperglicêmico.

Dessa forma, a via SC fica indicada em cães após a completa hidratação e controle glicêmico. Insulina R, na mesma dosagem, pode ser utilizada em intervalos de quatro, seis ou oito horas, até que o paciente volte a se alimentar e possa receber insulina NPH (0,5-2,2UI kg-1, SC, BID) (CHASTAIN, 1981, BOYSEN, 2008). Recentemente lançada no Brasil, a solução aquosa de insulina suína altamente purificada (composta por 30\% de insulina de zinco amorfa e 70\% de insulina de zinco cristalina - Caninsulin ${ }^{\circledR}$, Intervet) pode ser uma opção satisfatória para o controle glicêmico. Sua vantagem norteia-se no fato de poder ser administrada a cada 24 horas; no entanto, alguns estudos apontam que administração diária pode ser adequada somente para alguns pacientes. A maioria dos caninos requer duas aplicações diárias (HORN \& MITTEN, 2000; FLEEMAN et al., 2009). A figura 1 representa um diagrama comparativo das medidas terapêuticas adotadas nos casos de cetoacidose diabética e diabetes mellitus canina.

A hipocalemia é citada como potencial complicação em cães que apresentam vômito e anorexia. Faz-se necessária, portanto, a monitoração do potássio sérico durante o tratamento, pois este tende a diminuir com a terapia insulínica e com a correção da acidose metabólica (CHASTAIN, 1981; BOYSEN, 2008), em razão de a glicose carrear o potássio para o meio intracelular (BRUYETTE, 1997). A eletrocardiografia (ECG) pode ser útil na monitoração do paciente e estimativa da concentração de potássio sérico. Como sinais de hipocalemia, o ECG pode apresentar prolongamento do intervalo QT $(>0,22$ s em cães e $>0,16 \mathrm{~s}$ em gatos), bradicardia e onda $\mathrm{T}$ pequena e bifásica (CHASTAIN, 1981).

O uso do bicarbonato é muito controverso, pois pode exacerbar a hipocalemia e diminuir a distribuição do oxigênio para os tecidos, por aumentar a afinidade da hemoglobina pelo oxigênio (MACINTIRE, 1993, BOYSEN, 2008). Contudo, o bicarbonato pode ser administrado quando sua concentração sérica estiver inferior a 50\% da normal $\left(<12 \mathrm{mEq} \mathrm{L}^{-1}\right)$ ou quando o $\mathrm{pH}$ sanguíneo estiver inferior a 7,1 (CHASTAIN, 1981). Para a reposição de bicarbonato, o ideal é a monitoração pela hemogasometria, calculada pela fórmula [Bicarbonato 


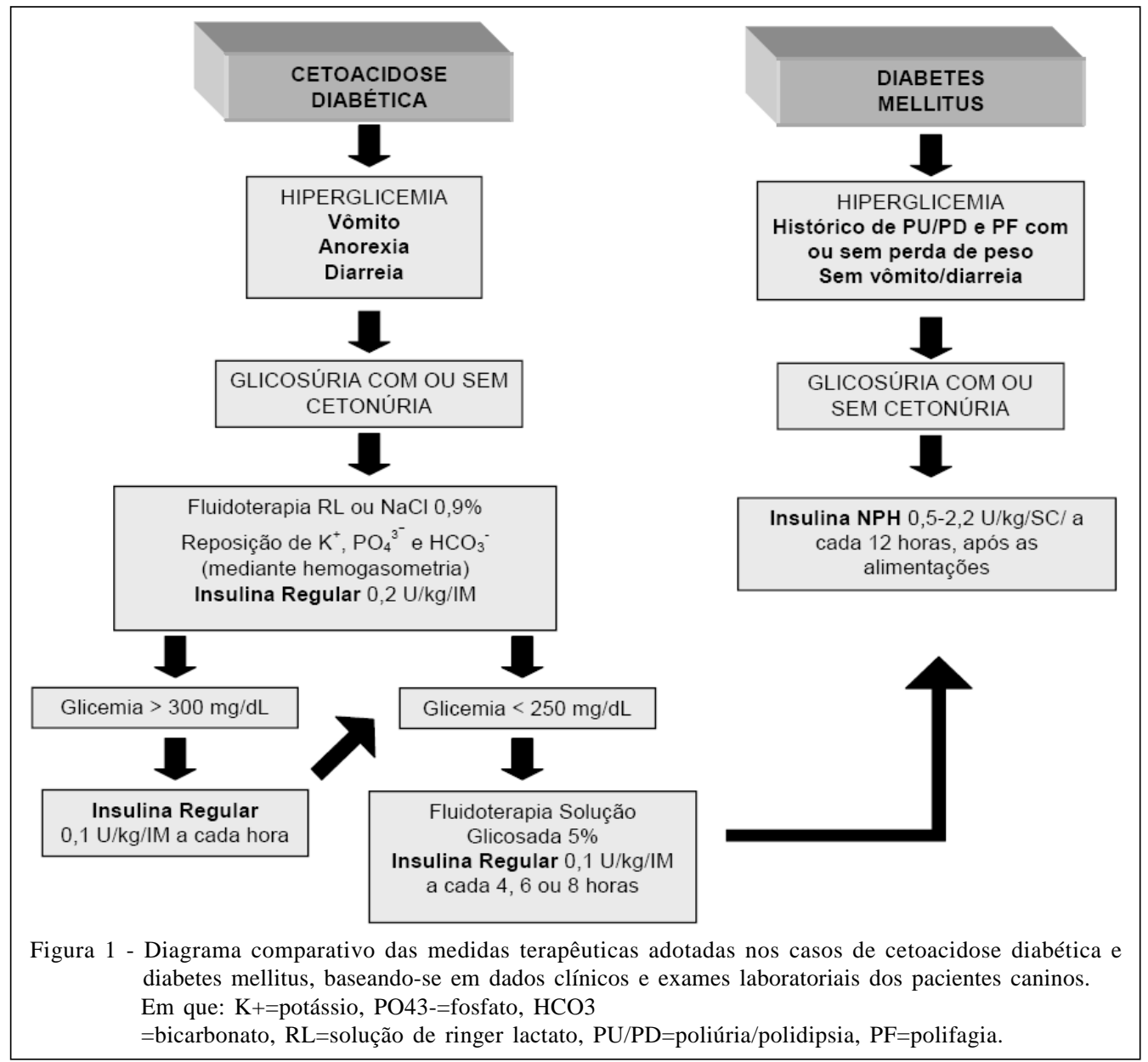

$(\mathrm{mEq})=$ Peso(kg) $\mathrm{x} 0,4 \mathrm{x}(24$ - bicarbonato paciente $) \mathrm{x} 0,5]$, em que o fator 0,5 indica metade da dose a ser infundida lentamente pela via intravenosa no período de seis horas (BRUYETTE, 1997). Estudos em humanos com CAD demonstraram que a administração de bicarbonato não traz benefícios em pacientes com $\mathrm{pH}$ sanguíneo entre 6,9 e 7,1 (VIALLON et al., 1999), além de levar ao aumento da cetogênese hepática (OKUDA et al., 1996). De acordo com BARONE et al. (2007), existem evidências de que a terapia com bicarbonato pode aumentar a síntese de corpos cetônicos, contrapondo-se, assim, aos efeitos da insulina. Portanto, quando não se tem acesso a hemogasometria, a administração empírica de bicarbonato só deve ser encorajada em paciente em coma, em que seja possível monitorar o potássio sérico e se consiga manter ventilação adequada.

O fosfato, geralmente associado ao fósforo, também pode ter sua concentração diminuída no tratamento da CAD, evidenciando sinais de disfunção plaquetária, diminuição da fagocitose, anormalidades neurológicas, hemólise, rabdomiólise, alterações cardíacas e falência respiratória (BRUYETTE, 1997). Na figura 1, compôs-se um diagrama contendo os principais pontos do tratamento da CAD canina, em comparação ao tratamento convencional do diabetes mellitus.

Como forma de acelerar a recuperação, podese optar pela alimentação por sonda nasogástrica, esofágica ou gástrica nos pacientes que não apresentarem mais episódios eméticos e a glicemia se mantiver próximo de $200 \mathrm{mg} \mathrm{dL}^{-1}$. O suporte nutricional enteral via sonda esofágica é uma boa opção em razão da fácil implantação e do fácil manejo, permitindo a passagem de alimento apropriado para diabéticos, sem impedir o animal de ingerir alimentos e água por conta própria (BRUNETTO et al., 2009).

O prognóstico da CAD é reservado, e o agravamento do quadro ocorre por doenças subjacentes (MACINTIRE, 1993). Destacam-se o hiperadrenocorticismo, a piometra, as hemoparasitoses, a insuficiência renal aguda, dentre outras (CHASTAIN, 1981; MACINTIRE, 1993; DUARTE et al., 2002; NELSON, 2006). 
O edema cerebral é descrito como uma das causas de morte durante o tratamento, gerado pelo abrupto declínio da glicemia ou por excesso de fluidos hipotônicos e hipocalemia, causados pelo uso inadequado de insulina e bicarbonato (CHASTAIN, 1981, BARONE et al., 2007). Dessa forma, o estabelecimento da correta insulinoterapia por meio da realização de curvas glicêmicas em pacientes com DM, assim como a monitoração de possíveis doenças desencadeantes da CAD, reduzem as chances de morte (NELSON, 2006).

Os proprietários dos pacientes diabéticos devem ser orientados a procurar atendimento médicoveterinário de urgência caso seu animal esteja vomitando ou inapetente. Salienta-se que não devem deixar de aplicar a dose de insulina rotineira por medo de causar hipoglicemia, tão pouco omitir do clínico alterações na posologia, pois falhas terapêuticas podem desencadear quadro grave de CAD em poucas horas (BARONE et al., 2007).

\section{CONCLUSÃO}

Tendo como base a fundamentação bibliográfica e buscando contribuir para um melhor entendimento da CAD, espera-se que, com esta revisão, os clínicos possam reconhecê-la e atuar mais rapidamente, utilizando protocolos terapêuticos mais seguros. Entretanto, ressalta-se que tão importante quanto um tratamento apropriado é a prevenção da $\mathrm{CAD}$, uma vez, que, na maioria das vezes, trata-se de uma complicação que pode ser prevenida pela orientação adequada aos proprietários desses pacientes.

\section{AGRADECIMENTOS}

À médica veterinária Mariana Cristina Hoeppner Rondelli, pela versão do resumo para a língua inglesa.

\section{REFERÊNCIAS}

BARONE, B. et al. Cetoacidose diabética em adultos atualização de uma complicação antiga. Arquivos brasileiros de endocrinologia e metabologia, Rio de Janeiro, v.51, n.9, p.1434-1447, 2007. Disponível em: <http://www.scielo.br/pdf/ abem/v51n9/03.pdf>. Acesso em: 5 jun. 2009.

BOYSEN, S.R. Fluid and electrolyte therapy in endocrine disorders: diabetes mellitus and hypoadrenocorticism. Veterinary Clinics of North America: Small Animal Practice, Philadelphia, v.38, p.699-717, 2008. Disponível em: <http://www.vetsmall.theclinics.com/article/S01955616(08)00003-X/pdf>. Acesso em 17 jun. 2009. doi:10.1016/ j.cvsm.2008.01.001.
BRUNETTO, M.A. et al. Suporte nutricional enteral no paciente crítico. Clínica Veterinária, São Paulo, n.78, p.40-49, 2009.

BRUYETTE, D.S. Diabetic ketoacidosis. Seminars in Veterinary Medicine and Surgery (Small Animal), Orlando, v.12, n.4, p239-247, 1997.

BUSH, B.M. Interpretação de resultados laboratoriais para clínicos de pequenos animais. Roca: São Paulo, 2004. 376p.

CHASTAIN, C.B. Intensive care of dogs and cats with diabetic ketoacidosis. Journal of the American Veterinary Medical Association, Ithaca, v.170, n.10, p.972-978, 1981.

CHASTAIN, C.B.; NICHOLS, C.E. Low-dose intramuscular insulin therapy for diabetic ketoacidosis in dogs. Journal of the American Veterinary Medical Association, Ithaca, v.178, n.6, p.561-564, 1981.

DE MARCO, V. et al. Diagnóstico de diabetes mellitus na espécie canina e avaliação a longo prazo da terapia insulínica através das concentrações sérica de hemoglobina glicosilada. Revista de Educação Continuada do CRMV-SP, São Paulo, v.2, p.2328, 1999. Disponível em: <http://www.hostcentral.com.br/crmv/ PDF/v2n2a05.pdf>. Acesso em: 25 jul. 2008.

DUARTE, R. et al. Accuracy of serum â-hydroxybutyrate managements for the diagnosis of diabetic ketoacidosis in 116 dogs. Journal of veterinary internal medicine, Philadelphia v.166, p.411-417, 2002. Disponível em: <http://doi.wiley.com/ 10.111//j.1939-1676.2002.tb01258.x>. Acesso em: 11 jun. 2009. doi: 10.1111/j.1939-1676.2002.tb01258.x.

FELDMAN, E.C.; NELSON, R.W. Diabetic ketoacidosis. In: Canine and feline endocrinology and reproduction. 2.ed. Philadelphia: Saunders, 1996. p.392421.

FLEEMAN, L.M. et al. Pharmacokinetics and pharmacodynamics of porcine insulin zinc suspension on eight diabetic dogs. Veterinary Record, London, v.164, n.8, p.232-237, 2009. Disponível em: <http://veterinaryrecord.bvapublications.com/cgi/ reprint/164/8/232>. Acesso em: 09 jun. 2009.

FOSS-FREITAS, M.C.; FOSS, M.C. Cetoacidose diabética e estado hiperglicêmico hiperosmolar. Simpósio: urgências e emergências endócrinas, metabólicas e nutricionais. Medicina, Ribeirão Preto, v.36, p.389-393, 2003

FOSTER, D.W.; McGARRY, J.D. The metabolic derangements and treatment of diabetic ketoacidosis. New England Journal of Medicine, Boston, n.309, p.159-169, 1983.

GRECO, D.S. Diagnosis of diabetes mellitus in cats and dogs. Veterinary Clinics of North America: Small Animal Practice, Philadelphia, v.31, n.5, p.845-853, 2001. Disponível em: <http://www.ncbi.nlm.nih.gov/pubmed/11570129>. Acesso em: 22 de jun. 2008.

HORN, B.; MITTEN, R.W. Evaluation of an insulin zinc suspension for control of naturally occurring diabetes mellitus in dogs. Australian veterinary journal, Sydney, v.78, n.12, p.831-834, 2000. Disponível em: <http://www.intervet.at/ binaries/108_122718.pdf>. Acesso em: 10 jun. 2009. 
HUME, D.Z. et al. Outcome of dogs with diabetic ketoacidosis: 127 dogs (1993-2003). Journal of veterinary internal medicine, Philadelphia, v.20, p.547-555, 2006. Disponível em: <http://www3.interscience.wiley.com/cgi-bin/fulltext/ 119820323/PDFSTART $>$. Acesso em: 11 jun. 2009. doi: 10.1111/j.1939-1676.2006.tb02895.x.

MACINTIRE, D.K. Treatment of diabetic ketoacidosis in dog by continuous low-dose intravenous infusion of insulin. Journal of the American Veterinary Medical Association, Ithaca, v.202, n.8, p.1266-1272, 1993.

McGUIRE, N.C. et al. Detection of occult urinary tract infections in dogs with diabetes mellitus. Journal of the American Animal Hospital Association, South Bend, v.38, p.541-544, 2002. Disponível em: <http://www.jaaha.org/cgi/ reprint/38/6/541>. Acesso em: 13 mai. 2008.

NELSON, R.W. Distúrbios do pâncreas endócrino. In: NELSON, R.W.; COUTO, C.G. Medicina interna de pequenos animais. 3.ed. Rio de Janeiro: Guanabara Koogan, 2006. p.701-743.

NOGUEIRA, R.M.B.; DE MARCO, V. Terapêutica das principais endocrinopatias em cães e gatos. In: ANDRADE, S.F. Manual de terapêutica veterinária. 3.ed. Roca: São Paulo. 2008. p.398-408.

OKUDA, Y. et al. Counterproductive effects of sodium bicarbonate in diabetic ketoacidosis. Journal of Clinical Endocrinology and Metabolism, Springfield, v.81, p.314320, 1996.

PINEDA, M.H.; DOOLEY, M.P. McDonald's veterinary endocrinology and reproduction. 5.ed. Iowa: Blackwell, 2003. 556p.
PÖPPL, A.G.; GONZÁLEZ, F.H.D. Aspectos epidemiológicos e clínico-laboratoriais da diabetes mellitus em cães. Acta Scientiae Veterinariae, Porto Alegre, v.33, n.1, p.33-40, 2005. Disponível em: <http://www.ufrgs.br/actavet/33-1/ artigo605.pdf $>$. Acesso em: 13 mai. 2008.

DI TOMMASO, M. et al. Evaluation of a portable meter to measure ketonemia and comparison with ketonuria for the diagnosis of canine diabetic ketoacidosis. Journal of veterinary internal medicine, Philadelphia, v.23, p.466-471, 2009. Disponível em: <http://www3.interscience.wiley.com/cgi-bin/ fulltext/122322398/PDFSTART>. Acesso em: 10 jun. 2009. doi: 10.1111/j.1939-1676.2009.0302.x.

UMPIERREZ, G.E. et al. Efficacy of subcutaneous insulin lispro versus continuous intravenous regular insulin for the treatment of patients with diabetic ketoacidosis. American journal of medicine, New York, v.17; p.291-296, 2004a. Disponível em: <http:// www.sciencedirect.com/science?_ob=MImg\&_imagekey=B6TDC4D5NNPG-1-3\&_cdi=5195\&_user=972052\&_orig=search\&_c overDate $=09 \% 2 F 01 \% 2 F 2004 \& \_s k=998829994 \& v i e w=c \& w c h p=d G L b V l b-$ zSkzS\&md5=f665259dbfc5c4546f53de791f324d41\&ie $=/$ sdarticle.pdf >. Acesso em: 14 mai. 2008 . doi:10.1016/ j.amjmed.2004.05.010.

UMPIERREZ, G.E. et al. Treatment of diabetic ketoacidosis with subcutaneous insulin aspart. Diabetes care, New York, v.27, n.8, p.1873-1878, 2004b. Disponível em: <http:// care.diabetesjournals.org/content/27/8/1873.full.pdf+html> . Acesso em: 14 mai. 2008.

VIALLON, A. et al. Does bicarbonate therapy improve the management of severe diabetic ketoacidosis? Critical Care Medicine, New York, v.27, n.12, p.2690-2693, 1999. 